Proc. XII Int. School on Theoretical Physics — Symmetry and Structural Properties of Condensed Matter

\title{
The Influence of Sonication and Silver Nanoparticles Doped on Viscoelastic Structure of Agarose Gel
}

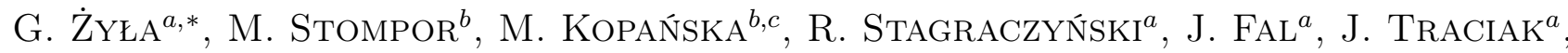 \\ M. Trybus ${ }^{a}$, S. Wolski $^{a}$, I. ZaWlik ${ }^{b, c}$, J. CeBulski $^{d}$, M. Konefat-Janocha $^{e}$ \\ AND M. ChOLEWA ${ }^{e}$ \\ ${ }^{a}$ Department of Physics and Medical Engineering, Rzeszów University of Technology, Rzeszów, Poland \\ ${ }^{b}$ Centre for Innovative Research in Medical and Natural Sciences, University of Rzeszów, Rzeszów, Poland \\ ${ }^{c}$ Department of Genetics, Chair of Molecular Medicine, University of Rzeszów, Rzeszów, Poland \\ ${ }^{d}$ Department of Experimental Physics, University of Rzeszów, Rzeszów, Poland \\ ${ }^{e}$ Department of Biophysics, University of Rzeszów, Rzeszów, Poland
}

\begin{abstract}
The paper presents result of experimental measurements of viscoelastic properties of agarose gel after sonication and with silver nanoparticles doped. Researches were conducted using a HAAKE MARS 2 rheometer (Thermo Electron Corporation, Karlsruhe, Germany), with serrated plate-plate measuring geometry. Viscoelastic properties of samples were measured with oscillation tests at constant deformation rate $0.1 \%$, and frequency $1 \mathrm{~Hz}$ in the temperature range from 278 to $348 \mathrm{~K}$. It was presented that using the sonication before solidification of gel results in increases of the storage modulus and complex viscosity of the solidified gel. It was also presented that when silver nanoparticles are doped into agarose gel, storage modulus and complex viscosity start to decrease at lower temperature.
\end{abstract}

DOI: 10.12693/APhysPolA.132.152

PACS/topics: 83.10.Tv, 83.60.Df, 83.80.Hj, 62.20.F-, 66.20.Ej

\section{Introduction}

Natural polymers have found large-scale application in the biomedical area [1]. Most of them are solid, have different mechanical and thermal strength and are biodegradable, which is their main advantage. Thus, there are attempts to use them in therapeutics, e.g. as coating of drugs. The possibility to modify biopolymers with the help of functional additives allows to improve their properties as packaging materials, which therefore gain some unique features [2]. Additionally, immobilization of biological material may be employed as a new method of oral administration of drugs and vaccines. Using polymer-based drug carriers ensures safe delivery of therapeutics and their release at the required site of action [3]. Natural polymers have attracted a great interest due to their bioacceptability and known degradation mechanisms, however, there are also some difficulties related to their poor biomechanical properties.

For production of hydrogel matrices there are natural compounds used. They gel by forming intermolecular hydrogen, hydrophobic or covalent bonds and also by ionic interactions. They have to meet some criteria: they should be non-toxic to the cells being immobilized, they should gel under mild temperature conditions and in non-toxic solvents and have proper mechanical properties.

Bactericidal properties of silver have been used in

\footnotetext{
*corresponding author; e-mail: gzyla@prz.edu.pl
}

medicine for years. There are several reports that confirm therapeutic effectiveness of nanosilver containing materials in orthopedics, surgery, or dermatology [4, 5]. Scald wound dressing that contain silver nanoparticles demonstrate longer antibacterial activity and better penetration into necrotic tissue compared to conventional metal compounds [6]. Moreover, using chitosan-encapsulated silver nanoparticles enhances their antibacterial activity [7]. The exceptional physicochemical properties of silver nanoparticles of less than $100 \mathrm{~nm}$ in diameter arise from the high surface area-to-volume ratio. Hence it is possible to achieve good antimicrobial activity at low concentrations of silver, which has been reported in literature. However, in situ synthesis of nanoparticles within the polysaccharide matrix may result in formation of the mixture of nanoparticles of different sizes, which may have some unwanted properties, because the activity of a nanoparticle depends mainly on its size, its active surface, and the ability to release silver ions [8].

In literature there have been mentioned many methods of preparation of silver nanoparticles. They include, among others, methods using colloidal silver nanoparticles, chemical reduction of silver salts, ultrasoundassisted syntheses and methods employing supercritical fluid technology $[9,10]$. The most commonly used source of silver ions in these methods is silver nitrate $(\mathrm{V})$.

Recently, researchers have focused their attention on development of new functional materials which contain nanoparticles dispersed in the gel matrix [11, 12]. Employment of natural cross-linking compounds ensures good bioavailability and biocompatibility of the obtained materials, as well as their low toxicity and full biodegrad- 
ability. Agarose is a well water-soluble polysaccharide derived from agar, which consists of galactose units and at room temperature forms a gel. Melting temperatures of such gels are quite high, and reach several dozen Celsius degrees. Being a porous carrier, the agarose gel finds wide application in laboratory diagnostics, mainly for separation of macromolecules, such as nucleic acids. There are also some disadvantages of agarose gels, which include their poor mechanical strength and difficulties with fixation after the separation.

The aim of this research was to develop a method of obtaining dispersion of silver nanoparticles of known size $(100 \mathrm{~nm})$ in a $2 \%$ agarose gel and to evaluate rheological properties of the obtained biocomposites. Because of the global problem of pathogen resistance to antibiotics, there is a constant search for new solutions. Preparation of novel bio-nanocomposites based on biodegradable polymers, containing silver nanoparticles with proven antibacterial properties and evaluation of their physical properties, especially viscoelastic ones, will broaden the knowledge about their possible application in various branches of industry. The obtained polymeric composites, consisting of agarose - a natural gelling substance isolated from cell walls of red algae (Gelidium, Gelidella, Pterocladia, Gracillaria, Ahnfeltia) - and silver nanoparticles, may find application as potential antibacterial and antifungal agents in future.

\section{Materials and methods}

\subsection{Materials and sample preparation}

To produce agarose gels a Agarose Basic (CAS: 901236-6; PanReac AppliChem, Darmstadt, Germany) was used. Destilled water specially pure used in experiments was provided by POCH (Avantor Performance Materials Poland, Gliwice, Poland), CAS: 7732-18-5. Silver nanopowder was produced by Sigma-Aldrich (SigmaAldrich, Saint Louis, USA), CAS: 7440-22-4. Provenance and purities of the used materials are listed in Table I.

Briefly, agarose hydrogels $(2 \%)$ were prepared by dissolving $0.4 \mathrm{~g}$ of agarose powder in $20 \mathrm{~mL}$ distilled water and heated in microwave until complete dissolution to transparency and immediately poured into gel plate and stored for 20-40 min at room temperature.

TABLE I

Provenance and purities of the used materials.

\begin{tabular}{l|l|c}
\hline \hline Product & \multicolumn{1}{|c|}{ Provenance } & Purity \\
\hline water & Avantor Performance Materials Poland & 1.000 \\
agarose & PanReac AppliChem & $>0.999$ \\
silver NP & Sigma-Aldrich & $>0.995$
\end{tabular}

\subsection{Measuring system}

Viscoelastic properties of samples were measured with HAAKE MARS 2 rheometer (Thermo Electron Corporation, Karlsruhe, Germany), with the minimum measurable torque of $0.5 \mu \mathrm{N} \mathrm{m}$. A plate-plate geometry with diameter of $35 \mathrm{~mm}$ was used. To avoid slippage between the sample and the rotor it was decided to use the serrated measuring geometry presented in Fig. 1. Samples were measured with oscillation tests with constant deformation rate $0.1 \%$, and frequency $1 \mathrm{~Hz}$ in the temperature range from 278 to $348 \mathrm{~K}$. A Peltier system coupled with a Phoenix 2 thermostat (Thermo Electron Corporation, Karlsruhe, Germany) was used to control temperature. Additionally, in order to ensure constant conditions of sample, measuring geometry was closed inside teflon solvent trap.

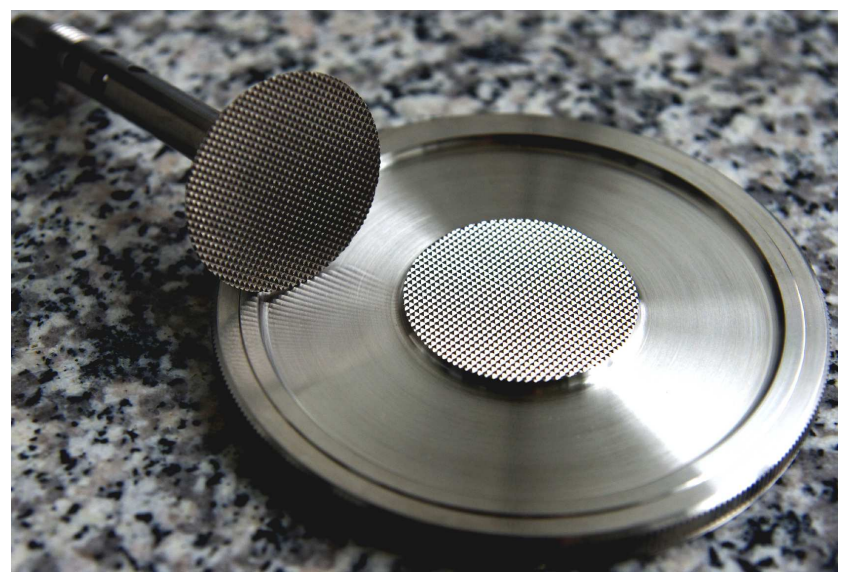

Fig. 1. Measuring geometry plate--plate $35 \mathrm{~mm}$ - serrated.

Prior to sample measurement geometry was heated. After the application of sample into the measuring geometry it was cooled to a temperature of $278 \mathrm{~K}$. After stabilization of the conditions inside the sample it was hold in a constant temperature for next $15 \mathrm{~min}$. Only after this time measurements of the viscoelastic properties were started.

\section{Results and discussion}

The study involved three types of gels. The first was a pure agarose gel, prepared as described in Sect. 2.1. The second sample was sonicated for $15 \mathrm{~min}$ in the VibraCell VCX130 (Sonics \& Materials, Inc., Newtown, USA) before measurements. In the process of preparing a third sample a silver nanoparticles with diameter less than $100 \mathrm{~nm}$ was added and suspension was sonicated prior measurements. The concentration of nanoparticles in gel was $1 \mathrm{mg} / \mathrm{mL}$.

Figure 2 presents results of measurements of storage modulus, and complex viscosity of various agarose gels at constant deformation rate $0.1 \%$, frequency $1 \mathrm{~Hz}$, and in temperature range from 278 to $334 \mathrm{~K}$.

It can be seen that the gel which was not treated with ultrasound before solidification has a lower value of storage modulus than gels that were sonicated during preparation process. The results also show that doped of silver nanoparticles in the agarose gel reduces the temperature at which the storage modulus starts decrease. 


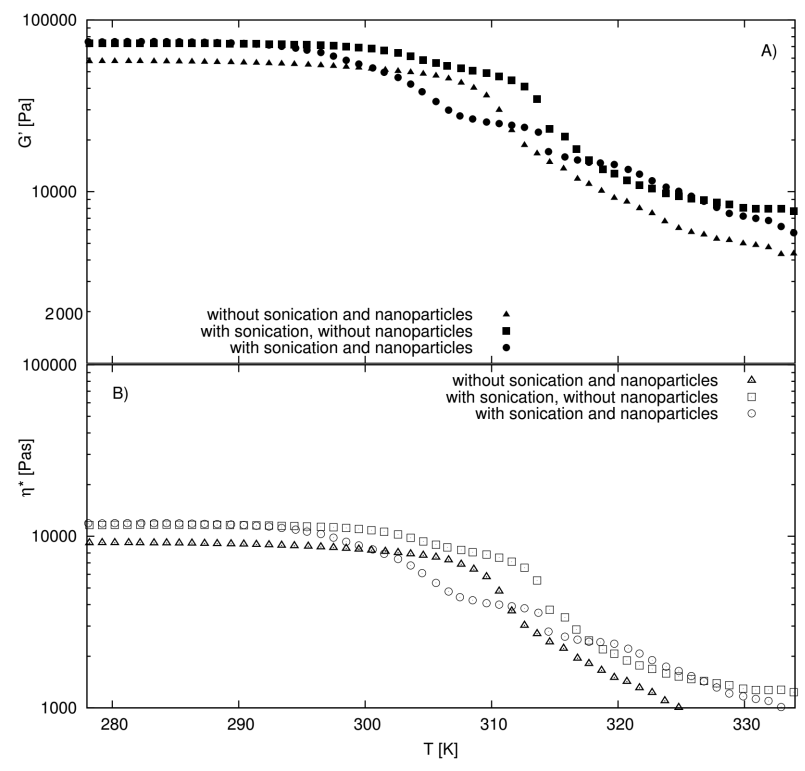

Fig. 2. Dependence of storage modulus (A), and complex viscosity (B) on temperature at constant deformation rate $0.1 \%$, and frequency $1 \mathrm{~Hz}$.
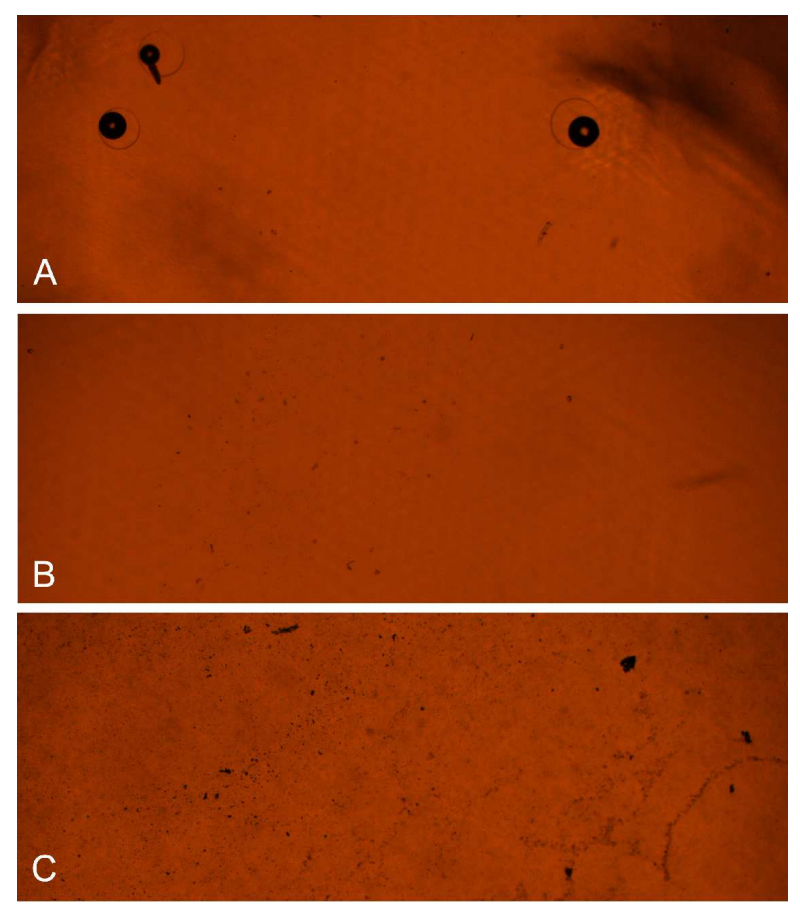

Fig. 3. Optical microscope pictures of gels (A) without sonication, (B) after sonication, (C) after silver nanoparticles doped, and sonicated.

Figure 3 presents pictures of measured gels, pictures was taken with polarizing microscope Eclipse LV100POL
(Nikon, Tokyo, Japan). In Fig. 3A we can see that air bubbles were created inside the gel, on the other hand, Fig. 3B presents that sonication process removed air bubbles from the material. This might be the reason of stronger viscoelastic structure of agarose gel as presented in Fig. 2. Figure 3C shows the gel after the addition of nanoparticles, as seen despite of sonication, it forms agglomerates when substance gelled.

\section{Conclusions}

Results of studies related to the effect of sonication during the production process of agarose gel on its viscoelastic properties was presented. It was presented that treating the gel with ultrasound enhances the elastic structure. This may be due to shaking of microbubbles of air from the gel, which was observed using a microscope. In addition, the effect of doping silver nanoparticles on storage modulus was measured. It shows that the addition of even very small quantities of silver nanoparticles leads to decrease the temperature at which there is a reduction of storage modulus.

\section{References}

[1] D. Feldman, J. Macromol. Sci. Part A Pure Appl. Chem. 53, 55 (2016).

[2] E. Malinowska-Pańczyk, K. Sztuka, I. Kołodziejska, Polimery 9, 625 (2010)

[3] L. Li, Z.-Y. He, X.-W. Wei, Regen Biomater. 3, 99 (2016).

[4] G. Franci, A. Falanga, S. Galdiero, L. Palomba, M. Rai, G. Morelli, M. Galdiero, Molecules 20, 8856 (2015).

[5] A. Mel, K. Chaloupka, Y. Malam, A. Darbyshire, B. Cousins, A.M. Seifalian, J. Biomed. Mater Res. 100A, 2348 (2012).

[6] N. Volkova, M. Yukhta, O. Pavlovich, A. Goltsev, Nano. Res. Lett. 11, 1 (2016).

[7] S. Agnihotri, S. Mukherji, S. Mukherji, Appl. Nanosci. 2, 179 (2012).

[8] X. Chen, H.J. Schluesener, Toxicol. Lett. 176, 1 (2008).

[9] G.C. Xu, J.J. Shi, D.J. Li, H.L. Xing, J. Polym. Res. 16, 295 (2009).

[10] J.J. Liu, Y.C. Yu, Q. Wu, J.C. Zhang, Chin. J. Inorg. Chem. 20, 321 (2004).

[11] X. Ma, Y. Xia, L. Ni, L. Song, Z. Wang, Spectrochim. Acta Part A Mol. Biomol. Spectrosc. 121, 657 (2014).

[12] Y.M. Mohan, K. Lee, T. Premkumar, K.E. Geckeler, Polymer 48, 158 (2007). 